\title{
Evolução das desigual dades sociais em saúde entre idosos e adultos brasileiros: um estudo baseado na Pesquisa Nacional por Amostra de Domicílios (PNAD 1998, 2003)
}

\section{Health inequality trends among Brazilian adults and old-aged: a study based on the National H ousehold Sample Survey (PNAD 1998, 2003)}

\author{
M aria Fernanda Lima-Costa ${ }^{1}$ \\ Divane Leite $M$ atos ${ }^{2}$ \\ Ana Amélia Camarano ${ }^{2}$
}

${ }^{1} \mathrm{~N}$ úcleo de Estudos em

Saúde Pública e

Envelhecimento da

Fundação Oswaldo Cruze

UniversidadeFederal de

Minas Gerais. Av. Augusto

de Lima 1715, 30190-002,

Belo Horizonte M G.

lima-costa@cpqrr.fiocruz.br

${ }^{2}$ Coordenação de População

eCidadania, I pea.
Abstract Theaim of this study was to verify whether health inequalities among Brazilian adults (20-64 years) and old-aged ( $\geq 65$ years) havechanged from 1998 to 2003. The study was conducted in samples of 203.455 and 239.700 participants of the National Household Sample Survey in 1998 and 2003 respectively. The health characteristics of thosein the lower quintile of the per capita family income were compared to those with higher incomeby means of multivariateanalysismethods. The characteristics considered in this study werehealth conditions and physical functioning, use of medical and dental services and health plan affiliation. The results from both years showed poorer health conditions, poorer physical functioning and lessuse of medical and dental services among those with lower per capita family incomein both agegroups, 20-64 and $\geq 65$ years. The associations between per capita family income, health status and use of health services weresi milar in 1998 and 2003, indicating that health inequalities havenot changed in the period under study. The persistence of these inequalities shows theinefficiency of public policies in the last five years to reduce such inequalities. Key words $\mathrm{H}$ eath inequalities, $\mathrm{H}$ ealth of theoldaged, $\mathrm{H}$ ealth of adults, PNAD
Resumo 0 objetivo destetrabalho foi verificar se as desigualdades sociais em saúde de adultos (2064 anos) eidosos ( $\geq 65$ anos) brasileiros se alteraram entre 1998 e 2003. 0 estudo foi realizado em uma amostra de 203.455 e 239.700 participantes da PNAD 1998 e 2003, respectivamente. As condições de saúde e função física, uso de serviços de saúde efiliação a plano de saúde daqueles pertencentes ao quintil inferior da distribuição da renda domiciliar per capita foram comparadasàs daqueles com renda mais alta, utilizando-se métodos multivariados deanálise. Osresultados mostraram que nos dois anos considerados, os indivíduos no estrato mais baixo de renda apresentavam piores condições de saúde, pi or função física emenor uso de serviços de saúde, tanto na faixa etária de 20 64 quanto na de $\geq 65$ anos de idade. As forças das associações entrerenda domiciliar per capita, condições de saúde e uso de serviços de saúde não se modificaram entre 1998 e 2003, indicando quenão houvealterações nas desigualdades sociaisem saúde no período estudado. A persistência dessas desigualdades aponta para a ineficiência de políticas, nos últimos cinco anos, que as reduzissem.

Palavras-chave D esigualdades sociais, Saúde dos idosos, Saúde dos adultos, PNAD 


\section{Introdução}

As desigualdades sociais vêm aumentando em todo o mundo, particularmente nos países em desenvolvimento. Estudo recente do Banco Mundial aponta para 0 aumento das desigualdades entre países, assim como dentro dos mesmos $^{1}$. O Brasil se coloca entre aqueles com um dos mais el evados graus de desigual dade social no mundo. Em paralelo, a população idosa experimenta um crescimento sem precedentes na história2, 3 . Atualmente, encontram-se ao redor do mundo 476 milhões de pessoas com 65 anos ou mais de idade (idosos), $61 \%$ das quais vivendo em regiões em desenvolvimento e 3,6 bilhões com idades compreendidas entre 20 a 65 anos4. A simultaneidade do envel hecimento populacional com o aumento das desigualdades sociais é um tema importante na agenda das políticas públicas nacionais e internacionais.

Entretanto, existem algumas controvérsias com relação à associação entre desigual dade social ou pobreza esaúde da população idosa5. Diversos estudos de base populacional, conduzidos em países desenvolvidos, mostraram que idosos com melhor situação socioeconômica apresentavam melhores condições de saúde ${ }^{6-13}$. Outros trabalhos, por outro lado, concluíram que a força dessa associação diminui ou mesmo desaparece nas idades mais velhas ${ }^{14-21}$. A redução ou desaparecimento dessa associação poderia ser explicada por: 1) viés de sobrevivência (indivíduos mais pobres tendem a morrer mais cedo); 2) exclusão de idosos institucionalizados (de uma maneira geral, estudos de base populacional não incluem idosos institucionalizados); 3) redução das diferenças de renda após a aposentadoria, com reflexos nas condições de saúde; 4) redução nas diferenças de acesso a serviços médicos de qualidade, devido a políticas para reduzir desigual dades sociais entreidosos, como o M edicare nos Estados Unidos ${ }^{11}$.

No Brasil, existem fortes evidências de que as condições de saúde da população idosa são influenciadas pela sua situação socioeconômi$\mathrm{ca}^{22,23}$. Existem, também, evidências de que a situação econômica afeta igualmente a saúde dos idosos e adultos brasileiros. Em um estudo, utilizando a base de dados da Pesquisa Nacional por Amostra de Domicílios (PNAD) conduzida em 1998, observou-se que indivíduos situados no estrato mais baixo de renda domiciliar per capita ( $<67 \%$ do valor do salário mínimo), independentemente da idade (20-64 ou maior ou igual a 65 anos), apresentavam piores condições de saúde, pior função física e uso menos fre- qüente deserviços de saúde. Os autores desse trabal ho levantaram a hipótese de que o uso menos freqüente de serviços de saúde, tanto por idosos quanto por adultos mais pobres, poderia estar contribuindo para a sua pior condição de saúde e chamaram a atenção para a gravidade desse achado, considerando-se que 0 acesso aos serviços médicos no Brasil é um direito constitucional24.

0 presente trabalho tem por objetivo verificar se as associações entre desigualdades sociais e saúde de adultos e idosos brasileiros foram alteradas entre 1998 e 2003. Para isso, foram utilizadas as bases de dados da Pesquisa Nacional por Amostra de Domicílios, conduzidas nos anos correspondentes.

\section{Metodologia}

\section{Pesquisa Nacional por Amostra de} Domicílios (PNAD)

As PNADS são realizadas anualmente, com exceção dos anos censitários, pela Fundação Instituto Brasileiro deGeografia estatística (IBGE), com o objetivo de obter informações sobre características demográficas, habitação, educação, trabalho e rendimentos da população brasileira. A pesquisa tem abrangência nacional, tendo incluído em 1998 e 2003 um suplemento com informações relativas a condições de saúde autoreferidas, uso de serviços de saúde e aspectos relacionados. Em 1998, a pesquisa foi realizada entre os dias 20 e 26 de setembro, e em 2003, entre 21 e 27 do mesmo mês ${ }^{25,26}$.

A PNAD baseia-se em uma amostra probabilística de domicílios, obtida em três estágios de seleção. No primeiro estágio, éfeita a seleção dos municípios, que são classificados como auto-representativos, com probabilidade 1 de pertencer à amostra; ecomo não auto-representativos, com probabilidade de pertencer à amostra proporcional à população residente. No segundo estágio, são selecionados os setores censitários, cuja probabilidade de inclusão é proporcional ao número de domicílios existentes no setor. No terceiro estágio, os domicílios são amostrados em cada setor censitário, sendo investigadas as informações relativas a todos os residentes no domicí$\operatorname{lio}^{25,26 .}$

\section{População estudada}

Todos os participantes da amostra da PNAD com idadeigual ou superior a 20 anos foram sele- 
cionados para o presentetrabalho, correspondendo a 203.455 indivíduos em 1998 e 239.700 em 2003. Neste trabalho, foram considerados idosos os participantes com 65 ou mais anos de idade. Como já mencionado anteriormente ${ }^{24}$, este ponto de corte foi adotado porque a literatura tem mostrado que a redução da força da associação (ou o seu desaparecimento) entre nível socioeconômico e saúde é observada a partir dessa idade.

\section{Variáveis}

\section{Variável dependente}

A variável dependente deste trabal ho éa renda domiciliar per capita, obtida a partir da renda total do domicílio dividida pelo número de moradores do mesmo. A renda dosidosos foi a referência para o cálculo da renda domiciliar per capita $^{24}$. Este procedimento foi adotado porque 0 foco do trabalho éa população idosa, eos domicílios com idosos residindo apresentam renda domiciliar per capita mais el evada do que os que não contam com idosos residindo ${ }^{27}$.

Os níveis de renda domiciliar per capita dos idosos foram agrupados em quintis e transformados em salários mínimos da época, como descrito em trabalho anterior ${ }^{24}$. Em 1998, o quintil inferior da distribuição da renda domiciliar per capita da população idosa foi de $R \$ 86,67$, correspondendo a $66,7 \%$ do salário-mínimo ( $R \$ 130,00$ no mês de setembro $)^{24}$. Em 2003, os valores correspondentes foram $R \$ 160,00$ e $66,7 \%$, respectivamente, considerando-se 0 valor do salário mínimo do mês de setembro de 2003 $(R \$ 240,00)$.

\section{Variáveis independentes}

Como variáveis independentes neste trabaIho, foram consideradas: 1) condições de saúdee função física - interrupção das atividades rotinei ras devido a problemas de saúde nas duas últimas semanas, ter estado acamado nas duas últimas semanas, presença e número de doenças crônicas auto-referidas, percepção da saúde e grau de dificuldade para realizar pelo menosuma entre três atividades da vida diária: alimentar-se, tomar banho ou ir ao banheiro; 2) uso de serviços de saúde - número de consultas médicas nos últimos 12 meses, tempo decorrido após a última visita ao dentista, número deinternações hospitalares nosúltimos 12 meses; e 3) filiação a plano de saúde.
Com referência às doenças crônicas auto-referidas, a pergunta adotada pela PNAD em 1998 foi "Tem a doença”. Em 2003, a pergunta adotada foi Algum médico ou profissional de saúde disse que vocêtem a doença $a^{25,26}$. 0 número de doenças crônicas foi obtido somando-seas respostas afirmativas às perguntas acima mencionadas. Com relação à percepção da saúde, em trabalho anterior, a informação foi desconsiderada quando outra pessoa respondeu à entrevista ${ }^{24}$. No presente trabalho, entretanto, levou-se em conta a informação para todos os participantes porque, recentemente, verificou-sequea inclusão das respostas dadas por outro respondente não alterava a distribuição da auto-avaliação da saúde, nem os fatores associados à mesma entre participantes da amostra da PNAD ${ }^{28}$.

\section{Análise dos dados}

A análise dos dados foi baseada em odds ratios e respectivos intervalos de confiança (método de Woolf), estimados por meio de regressão logísti$\mathrm{ca}^{29}$. Sexo, idade (variável contínua), respondente e macrorregião de residência foram consideradas a priori variáveis de confusão e foram incluídas em todos os modelos logísticos multivariados. A análise foi realizada, utilizando procedimentos para inquéritos populacionais do programa Stata ${ }^{30}$. Foram incorporados os pesos individuais (inverso da fração amostral) e correção para efeito do delineamento do estudo.

\section{Resultados}

Entre os 183.728 participantes da PNAD 1998 e os 215.639 da PNAD 2003 com 20-64 anos de idade, $97,0 \%$ e $97,7 \%$, respectivamente, foram incluídos no presente trabalho. As proporções correspondentes para a faixa etária de 65 anos ou mais foram $96,7 \%$ entre os 19.727 participantes da PNAD 1998, e 97,1\% entre os 24.061 participantes da de 2003. A idademédia das pessoas que tinham de 20 a 64 anos (adultos) em 1998 foi igual a 37,6 anos e 37,5 anos em 2003. Já a idade média dos idosos foi de 73,0 e 73,3 anos em 1998 e 2003, respectivamente.

$\mathrm{Na}$ tabela 1 estão apresentados os resultados da análise não ajustada da associação entre indicadores da condição de saúde e renda domiciliar per capita na fai xa etária de 20-64 anos, segundo 0 ano. Em ambos os períodos, observaram-seassociações significantes entrea renda domiciliar e a interrupção das atividades habituais nas duas 
últimas semanas por motivos de saúde, ter estado acamado nas duas últimas semanas, diabete auto-referido, percepção da saúde e grau de dificuldade para realizar pelo menos uma atividade da vida diária (exceto incapacidade total). Os demais indicadores apresentaram-se significativamente associados à renda domiciliar em 1998, mas não em 2003.

Com relação à faixa etária de 65 anos ou mais, observaram-se, em ambos períodos, associações significantes entre renda familiar e todos indicadores da condição de saúde investigados, exceto relato de artrite/reumatismo, hipertensão e número de doenças crônicas (Tabela 2).

Os resultados da análise não ajustada da associação entre ren da domiciliar per capita eindicadores de uso de serviços de saúde entre adultos e idosos estão apresentados nas tabelas 3 e 4 , respectivamente. Em ambos os grupos etários, tanto em 1998 quanto em 2003 foram observadas associações negativas entre renda domiciliar e filiação a plano de saúde, assim como número de consultas médicas nos últimos 12 meses. Associação positiva foi observada com tempo de-

Tabela 1

Análisenão ajustada da associação entre indicadores da condição de saúdee renda domiciliar per capita entre pessoas com 20 a 64 anos de idade (Brasil, 1998 e 2003) ${ }^{1}$.

\begin{tabular}{|c|c|c|c|c|c|c|}
\hline \multirow{4}{*}{ Indicadores } & \multicolumn{3}{|c|}{1998} & \multicolumn{3}{|c|}{2003} \\
\hline & \multicolumn{3}{|c|}{ Renda (em SM ) } & \multicolumn{3}{|c|}{ Renda (em SM) } \\
\hline & $<0,67$ & $>0,67$ & OR (IC 95\%) & $<0,67$ & $>0,67$ & OR (IC 95\%) \\
\hline & $\%$ & $\%$ & & $\%$ & $\%$ & \\
\hline $\begin{array}{l}\text { Interrupção das atividades habituais nas } \\
2 \text { últimas semanas por motivos de saúde }\end{array}$ & 8,3 & 5,9 & $1,46(1,36-1,56)$ & 8,3 & 6,0 & $1,44(1,35-1,53)$ \\
\hline Ter estado acamado nas 2 últimas semanas & 5,4 & 3,5 & $1,58(1,48-1,69)$ & 5,1 & 3,3 & $1,56(1,46-1,67)$ \\
\hline Artrite/reumatismo auto-referido & 13,6 & 8,9 & $1,62(1,50-1,75)$ & 6,9 & 6,7 & $1,04(0,97-1,11)$ \\
\hline Diabete auto-referido & 1,8 & 2,7 & $0,66(0,61-0,72)$ & 2,4 & 3,2 & $0,75(0,67-0,83)$ \\
\hline Hipertensão auto-referida & 14,8 & 14,2 & $1,05(1,01-1,10)$ & 14,9 & 15,5 & $0,95(0,90-1,00)$ \\
\hline \multicolumn{7}{|l|}{ N úmero de doen ças crôni cas auto-referidas } \\
\hline 0 & 70,7 & 74,0 & 1,00 & 75,5 & 74,0 & 1,00 \\
\hline 1 & 19,3 & 17,6 & $1,15(1,10-1,20)$ & 17,3 & 18,1 & $0,94(0,89-0,99)$ \\
\hline 2 & 7,1 & 5,9 & $1,25(1,17-1,34)$ & 5,2 & 5,5 & $0,93(0,87-1,00)$ \\
\hline $3+$ & 3,0 & 2,5 & $1,26(1,14-1,40)$ & 2,0 & 2,2 & $0,91(0,81-1,04)$ \\
\hline \multicolumn{7}{|l|}{ Percepção da saúde } \\
\hline M uito boa/boa & 66,7 & 77,1 & 1,00 & 67,7 & 78,8 & 1,00 \\
\hline Regular & 26,9 & 19,6 & $1,59(1,51-1,67)$ & 26,7 & 18,4 & $1,69(1,61-1,77)$ \\
\hline Ruim/muito ruim & 6,4 & 3,3 & $2,28(2,06-2,53)$ & 5,6 & 2,8 & $2,36(2,19-2,53)$ \\
\hline \multicolumn{7}{|l|}{$\begin{array}{l}\text { Grau de dificuldade para realizar pelo } \\
\text { menos uma atividade da vida diária }{ }^{2}\end{array}$} \\
\hline Nenhuma & 95,7 & 97,3 & 1,00 & 96,9 & 97,9 & 1,00 \\
\hline Pequena dificuldade & 3,0 & 2,0 & $1,61(1,43-1,81)$ & 2,6 & 1,4 & $1,49(1,34-1,66)$ \\
\hline Grande dificuldade & 1,0 & 0,6 & $1,70(1,46-1,99)$ & 0,8 & 0,5 & $1,56(1,39-1,76)$ \\
\hline Não consegue & 0,3 & 0,2 & $1,31(1,00-1,72)$ & 0,3 & 0,2 & $1,27(0,97-1,66)$ \\
\hline
\end{tabular}

${ }^{1}$ A nálise baseada na amostra de 178.229 pessoas 1998 e 210.716 pessoas em 2003.

${ }^{2}$ Alimentar-se, tomar banho ou ir ao banheiro.

SM : Salários mínimos da época (em 1998, cada = R\$130,00; em 2003, cada = R \$240,00)

OR (IC 95\%): O dds ratio não ajustado (intervalo de confiança em nível de 95\%)

$\%$ : percentagem de respostas afirmativas 
corrido após a última visita ao dentista. Entre adultos, a renda domiciliar apresentou associação positiva com hospitalizações em 1998 e 2003, mas essa associação não foi observada entre idosos.

Após ajustamentos por variáveis de confusão, todos os indicadores da condição geral de saúde (interrupção das atividades habituais nas 2 últimas semanas por motivos de saúde, ter estado acamado nas 2 últimas semanas e percepção de saúde) e a capacidade funcional (habilidadepara realizar atividades da vida diária) permaneceram significativa e positivamente associados à renda domiciliar per capita. Essas associações foram consistentemente observadas em adultos e em idosos, tanto em 1998 quanto em 2003. Com relação à morbidade auto-referida, os resultados diferiram nos dois grupos etários. Entre adultos, nos dois anos estudados, observaram-se associações significantes entre renda familiar e artrite/ reumatismo, hipertensão e número de doenças crônicas. Entre idosos, as associações encontradas não foram estatísticamente significantes em 1998 e/ou em 2003 (Tabela 5).

Tabela 2

Análise não ajustada da associação entre indicadores da condição de saúde e renda domiciliar per capita entre pessoas com 65 ou mais anos de idade (Brasil, 1998 e 2003) ${ }^{1}$.

\begin{tabular}{|c|c|c|c|c|c|c|}
\hline \multirow{4}{*}{ Indicadores } & \multicolumn{3}{|c|}{1998} & \multicolumn{3}{|c|}{2003} \\
\hline & \multicolumn{3}{|c|}{ Renda (em SM) } & \multicolumn{3}{|c|}{ Renda (em SM ) } \\
\hline & $<0,67$ & $>0,67$ & OR (IC 95\%) & $<0,67$ & $>0,67$ & OR (IC 95\%) \\
\hline & $\%$ & $\%$ & & $\%$ & $\%$ & \\
\hline $\begin{array}{l}\text { Interrupção das atividades habituais nas } \\
2 \text { últimas semanas por motivos de saúde }\end{array}$ & 17,2 & 14,5 & $1,23(1,08-1,39)$ & 17,0 & 13,3 & $1,33(1,24-1,43)$ \\
\hline Ter estado acamado nas 2 últimas semanas & 11,8 & 10,1 & $1,19(1,05-1,35)$ & 11,6 & 8,7 & $1,38(1,23-1,55)$ \\
\hline Artrite/reumatismo auto-referido & 46,2 & 38,7 & $1,36(1,24-1,49)$ & 29,2 & 29,7 & $0,98(0,91-1,06)$ \\
\hline Diabete auto-referido & 8,3 & 11,4 & $0,70(0,61-0,81)$ & 12,4 & 14,1 & $0,86(0,77-0,96)$ \\
\hline Hipertensão auto-referida & 44,6 & 45,9 & $0,95(0,87-1,04)$ & 50,1 & 51,3 & $0,95(0,86-1,06)$ \\
\hline \multicolumn{7}{|l|}{ Número de doenças crônicas auto-referidas } \\
\hline 0 & 26,9 & 28,4 & 1,00 & 31,7 & 29,1 & 1,00 \\
\hline 1 & 32,5 & 32,5 & $1,06(0,94-1,18)$ & 32,4 & 32,8 & $0,91(0,83-1,00)$ \\
\hline 2 & 23,7 & 22,5 & $1,11(0,99-1,26)$ & 22,5 & 22,9 & $0,90(0,80-1,02)$ \\
\hline $3+$ & 16,9 & 16,6 & $1,08(0,91-1,29)$ & 13,4 & 15,2 & $0,81(0,70-0,93)$ \\
\hline \multicolumn{7}{|l|}{ Percepção da saúde } \\
\hline Muito boa/boa & 27,8 & 37,8 & 1,00 & 30,4 & 42,7 & 1,00 \\
\hline Regular & 47,4 & 45,1 & $1,43(1,26-1,62)$ & 48,1 & 43,7 & $1,54(1,38-1,72)$ \\
\hline Ruim/muito ruim & 24,8 & 17,0 & $1,99(1,69-2,34)$ & 21,6 & 13,6 & $2,23(1,91-2,61)$ \\
\hline \multicolumn{7}{|l|}{$\begin{array}{l}\text { Grau de dificuldade para realizar pelo } \\
\text { menos uma atividade da vida diária }{ }^{2}\end{array}$} \\
\hline Nenhuma & 76,8 & 82,9 & 1,00 & 78,9 & 84,5 & 1,00 \\
\hline Pequena dificuldade & 12,6 & 9,6 & $1,41(1,20-1,66)$ & 9,8 & 8,2 & $1,28(1,13-1,45)$ \\
\hline Grande dificuldade & 7,5 & 5,0 & $1,61(1,34-1,94)$ & 6,5 & 4,6 & $1,50(1,28-1,75)$ \\
\hline Não consegue & 3,2 & 2,5 & $1,36(1,07-1,73)$ & 4,8 & 2,7 & $1,96(1,65-2,33)$ \\
\hline
\end{tabular}

${ }^{1}$ Análise baseada na amostra de 19.068 pessoas em 1998 e 23.364 pessoas em 2003.

${ }^{2}$ Alimentar-se, tomar banho ou ir ao banheiro.

SM : Salários mínimos da época (em 1998, cada = R\$130,00; em 2003, cada = R\$240,00).

OR (IC 95\%): O dds ratio não ajustado (intervalo de confiança em nível de 95\%).

$\%$ : percentagem de respostas afirmativas. 
$\mathrm{Na}$ tabela 6 estão apresentados os resultados da análise ajustada da associação entre indicadores de uso de serviços de saúde, assim como da filiação a plano de saúde, e renda domiciliar per capita. Associações negativas foram encontradas para filiação a plano de saúde e número de consultas médicas nos últimos 12 meses, e associação positiva foi encontrada para tempo decorrido após a última visita ao dentista. Esse padrão foi observado deforma consistentenos dois anos estudados, tanto em adultos quanto em idosos. Com relação às hospitalizações nos últimos12 meses, observaram-se diferenças entre adultos e idosos. Entre os primeiros, foi observada associação positiva entre hospitalizações e ren da familiar. Entre os últimos, as associações observadas não foram estatísticamente significantes, exceto para duas ou mais hospitalizações no ano de 2003.

\section{Discussão}

0 presente trabalho foi desenvolvido para responder a três perguntas. A primeira foi se as desigualdades sociais em saúde entre adultos e idosos brasileiros, observadas em 1998, persistiram cinco anos depois; a segunda é se a magnitude dessas desigualdades em 2003 foi semel hantenos dois grupos etários; e a terceira, se houve modificações na magnitude dessas desigual dades durante o período estudado. Como medida de desigualdade, optou-se por utilizar a renda domiciliar per capita situada no quintil inferior da

Tabela 3

Análise não ajustada da associação entre indicadores de uso de serviços de saúde e filiação a plano de saúde e renda domiciliar per capita entre pessoas com 20 a 64 anos de idade (Brasil, 1998 e 2003) ${ }^{1}$.

\begin{tabular}{|c|c|c|c|c|c|c|}
\hline \multirow{4}{*}{ Indicadores } & \multicolumn{3}{|c|}{1998} & \multicolumn{3}{|c|}{2003} \\
\hline & \multicolumn{3}{|c|}{ Renda (em SM) } & \multicolumn{3}{|c|}{ Renda (em SM) } \\
\hline & $<0,67$ & $>0,67$ & OR (IC 95\%) & $<0,67$ & $>0,67$ & OR (IC 95\%) \\
\hline & $\%$ & $\%$ & & $\%$ & $\%$ & \\
\hline Filiação a plano de saúde & 4,4 & 36,3 & $0,08(0,07-0,09)$ & 3,9 & 61,4 & $0,09(0,08-0,10)$ \\
\hline \multicolumn{7}{|l|}{$\begin{array}{l}\text { Tempo decorrido após a última } \\
\text { visita ao dentista (anos) }\end{array}$} \\
\hline$<1$ & 20,6 & 39,0 & 1,00 & 27,1 & 46,4 & 1,00 \\
\hline $1-2$ & 17,2 & 24,2 & $1,35(1,28-1,42)$ & 20,4 & 24,2 & $1,44(1,39-1,50)$ \\
\hline $3+$ & 52,8 & 35,5 & $2,90(2,67-3,15)$ & 44,6 & 27,6 & $2,77(2,59-2,97)$ \\
\hline Nunca visitou o dentista & 9,4 & 2,3 & $7,81(6,84-8,92)$ & 7,9 & 1,8 & $7,34(6,45-8,36)$ \\
\hline \multicolumn{7}{|l|}{ Número de consultas médicas nos } \\
\hline últimos 12 meses & 48,7 & 40,9 & 1,00 & 41,8 & 33,1 & 1,00 \\
\hline 0 & 15,3 & 16,4 & $0,79(0,75-0,83)$ & 16,1 & 17,5 & $0,73(0,69-0,76)$ \\
\hline 1 & 11,8 & 13,6 & $0,73(0,69-0,77)$ & 13,3 & 15,5 & $0,68(0,65-0,71)$ \\
\hline 2 & 24,1 & 29,1 & $0,70(0,66-0,74)$ & 28,8 & 33,8 & $0,68(0,63-0,73)$ \\
\hline $3+$ & & & & & & \\
\hline
\end{tabular}

Número de internações hospitalares nos últimos 12 meses

\begin{tabular}{lcccccc}
0 & 90,4 & 92,9 & 1,00 & 91,3 & 93,2 & 1,00 \\
1 & 7,6 & 5,8 & $1,35(1,28-1,43)$ & 6,9 & 5,5 & $1,28(1,19-1,37)$ \\
$2+$ & 2,0 & 1,3 & $1,52(1,38-1,67)$ & 1,7 & 13,2 & $1,33(1,20-1,48)$ \\
\hline
\end{tabular}

\footnotetext{
${ }^{1}$ Análise baseada na amostra de 178.229 pessoas 1998 e 210.716 pessoas em 2003.

SM : Salários mínimos da época (em 1998, cada = R\$130,00; em 2003, cada $=R \$ 240,00)$.

OR (IC 95\%): Odds ratio não ajustado (intervalo de confiança em nível de $95 \%$ ).

$\%$ : percentagem de respostas afirmativas.
} 
distribuição da renda, em comparação aos valores mais altos. Os resultados mostraram que as associações entre menor renda domiciliar per capita e piores condições gerais de saúde, menor capacidade funcional e menor uso de serviços médicos (consultas médicas) e odontológicos, que já haviam sido observadas em $1998^{24}$, persistem em 2003, tanto em adultos quanto em idosos.

Com relação à comparação entre adultos e idosos, com base nos dados da PNAD 2003, observou-sequeas forças das associações entre rendadomiciliar per capita eos indicadores das condições gerais de saúde, a capacidade funcional e os indicadores de uso de serviços de saúde apresentaram uma discreta ten dência a serem menores entre os idosos, em relação aos mais jovens. M as a direção das associações observadas foi a mesma e, na maioria das vezes, os intervalos de confiança dos odds ratios apresentaram-sesuperpostos, como já havia sido observado em trabaIho anterior, baseado nos dados da PNAD de $1998^{24}$. Por outro lado, com relação às doenças crônicas e à filiação a plano de saúde, as diferenças entre adultos e idosos foram mais expressivas. 0 relato de artrite/reumatismo e de hipertensão, assim como o número de doenças crônicas, foi mais freqüente entre adultos com pior nível socioeconômico, mas não entre os idosos. A força da associação entre renda familiar efiliação a plano de saúde foi mais forte entre os últimos do que entre os primeiros.

Com relação às tendências das desigualdades sociais em saúde entre 1998 e 2003, verificou-se que os resultados observadosem 1998 repetiramse em 2003, com raríssimas exceções, que são

Tabela 4

Análise não ajustada da asso ciação entre indicadores de uso de serviços de saúde e filiação a plano de saúde e renda domiciliar per capita entre pessoas com 65 ou mais anos de idade (Brasil, 1998 e 2003) ${ }^{1}$.

\begin{tabular}{|c|c|c|c|c|c|c|}
\hline \multirow{4}{*}{ Indicadores } & \multicolumn{3}{|c|}{1998} & \multicolumn{3}{|c|}{2003} \\
\hline & \multicolumn{3}{|c|}{ Renda (em SM) } & \multicolumn{3}{|c|}{ Renda (em SM) } \\
\hline & $<0,67$ & $>0,67$ & OR (IC 95\%) & $<0,67$ & $>0,67$ & OR (IC 95\%) \\
\hline & & & & $\%$ & $\%$ & \\
\hline Filiação a plano de saúde & 4,7 & 30,8 & $0,11(0,09-0,14)$ & 6,7 & 34,5 & $0,14(0,11-0,17)$ \\
\hline \multicolumn{7}{|l|}{$\begin{array}{l}\text { Tempo decorrido após a última } \\
\text { visita ao dentista (anos) }\end{array}$} \\
\hline$<1$ & 4,7 & 12,4 & 1,00 & 6,6 & 17,3 & 1,00 \\
\hline $1-2$ & 4,0 & 9,2 & $1,16(0,91-1,46)$ & 5,8 & 10,1 & $1,50(1,25-1,79)$ \\
\hline $3+$ & 77,4 & 73,2 & $2,78(2,24-3,44)$ & 74,7 & 67,7 & $2,88(2,49-3,33)$ \\
\hline Nunca visitou o dentista & 13,8 & 5,2 & $7,01(5,26-9,34)$ & 12,9 & 4,9 & $6,93(5,21-9,22)$ \\
\hline \multicolumn{7}{|c|}{$\begin{array}{l}\text { Número de consultas médicas nos } \\
\text { últimos } 12 \text { meses }\end{array}$} \\
\hline 0 & 35,3 & 24,5 & 1,00 & 26,4 & 19,0 & 1,00 \\
\hline 1 & 14,8 & 13,4 & $0,77(0,68-0,88)$ & 12,4 & 12,1 & $0,74(0,64-0,84)$ \\
\hline 2 & 13,3 & 14,2 & $0,64(0,56-0,74)$ & 13,2 & 14,5 & $0,65(0,56-0,77)$ \\
\hline $3+$ & 36,6 & 47,8 & $0,53(0,48-0,59)$ & 48,0 & 54,4 & $0,63(0,56-0,71)$ \\
\hline \multicolumn{7}{|l|}{$\begin{array}{l}\text { Número de internações } \\
\text { hospitalares nosúltimos } 12 \text { meses }\end{array}$} \\
\hline 0 & 85,3 & 85,1 & 1,00 & 85,4 & 86,0 & 1,00 \\
\hline 1 & 9,4 & 10,1 & $0,93(0,82-1,05)$ & 9,2 & 9,5 & $0,97(0,87-1,09)$ \\
\hline $2+$ & 5,3 & 4,9 & $1,09(0,87-1,35)$ & 5,4 & 4,5 & $1,22(1,06-1,41)$ \\
\hline
\end{tabular}

\footnotetext{
${ }^{1}$ Análise baseada na amostra de 19.068 pessoas em 1998 e 23.364 pessoas em 2003. SM : Salários mínimos da época (em 1998, cada = R \$130,00; em 2003, cada = R\$240,00). OR (IC 95\%): O dds ratio não ajustado (intervalo de confiança em nível de 95\%). $\%$ : percentagem de respostas afirmativas.
} 
pontuais e circunscritas a al gumas doenças crônicas auto-referidas. Essas semel hanças incluem todos os indicadores da condição geral de saúde, capacidadefuncional eindicadores deuso deserviços. A semelhança dos resultados obtidos em 1998 e em 2003 é de tal ordem, que a magnitude das associações entre renda domiciliar per capita e as variáveis estu dadas não se modificou durante o período em estudo.

O suplemento de saúde da PNAD representa a mais abrangente fonte de dados sobre as condições de saúde - e aspectos relacionados - da população brasileira. A sua periodicidade é im- portante para que se possam avaliar as tendências dessas condições e alguns dos seus determinantes. 0 presente trabalho é um dos muitos exemplos do uso de suas informações para estudar essas tendências. Os resultados deste trabaIho mostraram uma grande consistência entreos dados obtidos em 1998 e 2003, reforçando a sua utilidade para o estudo dessas tendências. Por outro lado, é importante chamar a atenção para o fato de que a renda domiciliar na PNAD possa estar subestimada, sobretudo nos estratos sociais mais altos, como já foi anteriormente comentado $^{24}$. Isto pode ter levado a um viés de classifi-

Tabela 5

Análise ajustada da associação entre indicadores da condição de saúde e renda domiciliar per capita, segundo a faixa etária (Brasil, 1998 e 2003) ${ }^{1}$.

\begin{tabular}{|c|c|c|c|c|}
\hline \multirow{2}{*}{ Indicadores } & \multicolumn{2}{|c|}{20 a 64 anos } & \multicolumn{2}{|c|}{65 an os ou mais } \\
\hline & $\begin{array}{c}1998 \\
\text { OR (IC 95\%) }\end{array}$ & $\frac{2003}{\text { OR (IC 95\%) }}$ & $\begin{array}{c}1998 \\
\text { OR (IC } 95 \%)\end{array}$ & $\begin{array}{c}2003 \\
\text { OR (IC } 95 \%)\end{array}$ \\
\hline $\begin{array}{l}\text { Interrupção das atividades habituais nas } \\
2 \text { últimas semanas por motivos de saúde }\end{array}$ & $1,41(1,32-1,51)$ & $1,47(1,40-1,55)$ & $1,21(1,07-1,37)$ & $1,33(1,23-1,43)$ \\
\hline Ter estado acamado nas 2 últimas semanas & $1,55(1,45-1,65)$ & $1,60(1,51-1,69)$ & $1,20(1,05-1,36)$ & $1,39(1,24-1,57)$ \\
\hline Artrite/reumatismo auto-referido & $1,61(1,51-1,71)$ & $1,18(1,13-1,23)$ & $1,19(1,09-1,30)$ & $0,96(0,90-1,03)$ \\
\hline Diabete auto-referido & $0,83(0,75-0,91)$ & $0,99(0,91-1,09)$ & $0,78(0,68-0,90)$ & $0,94(0,85-1,04)$ \\
\hline Hipertensão auto-referida & $1,21(1,16-1,27)$ & $1,23(1,17-1,28)$ & $1,02(0,93-1,11)$ & $1,03(0,93-1,13)$ \\
\hline \multicolumn{5}{|l|}{ N úmero de doenças crônicas auto-referidas } \\
\hline 0 & 1,00 & 1,00 & 1,00 & 1,00 \\
\hline 1 & $1,27(1,22-1,33)$ & $1,13(1,09-1,18)$ & $1,03(0,93-1,15)$ & $0,97(0,89-1,05)$ \\
\hline 2 & $1,51(1,41-1,61)$ & $1,27(1,19-1,35)$ & $1,13(1,00-1,27)$ & $1,01(0,90-1,12)$ \\
\hline $3+$ & $1,63(1,48-1,80)$ & $1,38(1,24-1,53)$ & $1,13(0,96-1,33)$ & $0,95(0,84-1,06)$ \\
\hline \multicolumn{5}{|l|}{ Percepção da saúde } \\
\hline Muito boa/boa & 1,00 & 1,00 & 1,00 & 1,00 \\
\hline Regular & $1,66(1,57-1,74)$ & $1,82(1,71-1,93)$ & $1,33(1,19-1,49)$ & $1,40(1,27-1,54)$ \\
\hline Ruim/muito ruim & $2,50(2,27-2,75)$ & $2,74(2,54-2,96)$ & $1,70(1,48-1,95)$ & $1,90(1,66-2,17)$ \\
\hline \multicolumn{5}{|l|}{$\begin{array}{l}\text { Grau de dificuldade para realizar pelo } \\
\text { menos uma atividade da vida diária }{ }^{2}\end{array}$} \\
\hline Nenhuma & 1,00 & 1,00 & 1,00 & 1,00 \\
\hline Pequena dificuldade & $1,62(1,47-1,80)$ & $1,50(1,08-2,09)$ & $1,38(1,18-1,60)$ & $1,98(1,66-2,36)$ \\
\hline Grande dificuldade & $1,91(1,64-2,23)$ & $1,79(1,56-2,05)$ & $1,61(1,34-194)$ & $1,50(1,31-1,71)$ \\
\hline Não consegue & $1,62(1,23-2,12)$ & $1,64(1,48-1,81)$ & $1,45(1,13-1,87)$ & $1,33(1,18-1,50)$ \\
\hline
\end{tabular}

${ }^{1}$ Análise baseada em 178.229 pessoas com 20-64 anos e 19.068 participantes com 65 ou mais anos de idade em 1998; os dados correspondentes para 2003 são 210.716 e 23.364 , respectivamente.

${ }^{2}$ Alimentar-se, tomar banho ou ir ao banheiro.

OR (IC 95\%): O dds ratios (intervalo de confiança em nível de 95\%) ajustados pelo método de regressão logística múltipla por sexo, idade, macrorregião deresidência erespondente.

$\%$ : percentagem de respostas afirmativas. 
cação, reduzindo a força das associações encontradas. 0 viés de sobrevivência (indivíduos com menor renda apresentam menor sobrevida) também pode ter contribuído para diminuir as forças das associações entre nível socioeconômico e saúde, sobretudo entre os idosos ${ }^{24}$.

Em resumo, os resultados deste trabal ho mostraram que: 1) nos dois anos considerados, os indivíduos no estrato mais baixo de renda apresentavam piores condições de saúde, pior função física e menor uso de serviços de saúde, tanto na faixa etária de 20-64 quanto na de $>65$ anos de idade, mostrando que as desigualdades sociais afetam igualmente as condições de saúde de adultos e idosos neste país; 2) em ambos os períodos, essas desigualdades foram mais evidentes em relação ao uso de serviços de saúde, indicadores gerais da condição de saúde e capacidade funcional, que apresentam maior impacto sobre a qualidade de vida relacionada à saú de, do que em relação a doenças crônicas e/ou ao número dessas doenças; 3) as forças das associações entre renda familiar per capita, condições de saúdee uso de serviços de saúde não se modificaram entre 1998 e 2003 em adultos eidosos, indicando que não houve alterações nas desigualdades sociais em saúde no período estudado. A persistência dessas desigualdades aponta para a ineficiência de políticas nos últimos cinco anos que as reduzissem. Os resultados sugerem que medidas para a redução das desigual dades nas condições de saúde da população brasileira devem ir além das medidas de saúde pública, sem esquecer estas. Elas devem passar, também, pela diminuição das desigualdades sociais. 0 ponto que se coloca não é apenas a preocupação com a redução da mortalidade mas, também, com uma melhor qualidade de vida e saúde para adultos e idosos.

Tabela 6

Análise ajustada da associação entre indicadores de uso de serviços de saúde e filiação a plano de saúde e renda domiciliar per capita, segundo a faixa etária (Brasil, 1998 e 2003) ${ }^{1}$.

\begin{tabular}{|c|c|c|c|c|}
\hline \multirow{3}{*}{ Indicadores } & \multicolumn{2}{|c|}{20 a 64 anos } & \multicolumn{2}{|c|}{65 anos ou mais } \\
\hline & 1998 & 2003 & 1998 & 2003 \\
\hline & OR (IC 95\%) & OR (IC 95\%) & OR (IC 95\%) & OR (IC 95\%) \\
\hline Filiação a plano de saúde & $0,09(0,09-0,10)$ & $0,11(0,10-0,12)$ & $0,14(0,11-0,17)$ & $0,17(0,14-0,20)$ \\
\hline \multicolumn{5}{|c|}{$\begin{array}{l}\text { Tempo decorrido após a última visita ao } \\
\text { dentista (anos) }\end{array}$} \\
\hline$<1$ & 1,00 & 1,00 & 1,00 & 1,00 \\
\hline $1-2$ & $1,38(1,32-1,45)$ & $1,47(1,42-1,53)$ & $1,14(0,90-1,45)$ & $1,49(1,23-1,81)$ \\
\hline $3+$ & $3,22(3,01-3,45)$ & $3,19(3,06-3,33)$ & $2,58(2,10-3,17)$ & $2,64(2,29-3,05)$ \\
\hline Nunca visitou o dentista & $6,39(5,53-7,38)$ & $6,04(5,41-6,75)$ & $5,20(3,96-6,84)$ & $5,11(3,77-6,92)$ \\
\hline
\end{tabular}

Número de consultas médicas nos últimos 12 meses
0
1
1,00
1,00
1,00
1,00
2
$0,76(0,72-0,80) \quad 0,73(0,70-0,76)$
$0,71(0,67-0,75) \quad 0,68(0,65-0,71)$
$0,81(0,71-0,93) \quad 0,78(0,68-0,89)$
$3+$
$0,71(0,66-0,75)$
$0,71(0,67-0,75)$
$0,69(0,60-0,79) \quad 0,71(0,61-0,83)$
$0,65(0,59-0,73) \quad 0,75(0,68-$
$\mathrm{N}$ úmero de internações hospitalares nos
0
1
1,00
1,00
1,00
1,00
$1,31(1,25-1,37) \quad 1,25(1,18-1,33) \quad 0,95(0,84-1,07) \quad 0,97(0,87-1,08)$
$2+$
$1,61(1,47-1,77) \quad 1,37(1,27-1,48) \quad 1,16(0,94-1,44) \quad 1,24(1,10-1,39)$ últimos 12 meses

\footnotetext{
${ }^{1}$ Análise baseada em 178.229 pessoas com 20-64 anos e 19.068 participantes com 65 ou mais anos de idade em 1998; os dados correspondentes para 2003 são 210.716 e 23.364, respectivamente.

OR (IC 95\%): O dds ratios (intervalo de confiança em nível de 95\%) ajustados pelo método de regressão logística múltipla por sexo,

idade, macrorregião de residência e respondente.

$\%$ : percentagem de respostas afirmativas.
} 


\section{Colaboradores}

M F Lima-Costa delineou o estudo, orientou a análise dos dados, participou da discussão dos resultados e da redação do manuscrito. DL Matos realizou a análise dos dados, participou da discussão dos resultadose da redação final do manuscrito. AA Camarano participou da discussão dos resultados e da redação do manuscrito.

\section{Referências}

1. Banco Mundial. Relatório sobre o desenvolvimento mundial 2006. Eqüidade e desenvolvimento: visão geral. Washington, DC; 2006.

2. World Health Organization. Populaton ageing: public health challenge. Washington, DC: WHO; 1998. (WHO Fact Sheet No. 135).

3. World Health Organization. World Health Organization launches new initiative to address the health needs of a rapidly ageing population [cited 2005 N ovember 4]. Available from: http://www.who.int/mediacentre/news/releases/2004/pr60/en/print.htm

4. United Nations. Population Division of the Department of Economic and Social Affairs of the United Nations Secretariat. World population prospects: the 2004 revision and world urbanization prospects: the 2003 revision [cited 2006 Fev 20]. Available from: http://esa.un.org/unpp

5. Jefferys $M$. Social inequalities in health - do they diminish with age? [editorial]. Am J Public H ealth 1996; 86(4):474-5.

6. Maddox GL, Clark D O. Trajectories of functional impairment in later life. J Health Soc Behav 1992; 33(2):114-25.

7. Cairney J, Arnold R. Social class, health and aging: socioeconomic determinants of self-reported morbidity among noninstitutionalized elderly in Canada. Can J Public Health 1996; 87(3):199-203.

8. Robert S, House JS. SES differentials in health by age and alternative indicators of SES. J Aging H ealth 1996; 8(3):359-88.

9. Ross CE, Wu CL. Education, age, and the cumulative advantage in health. J Health Soc Behav 1996; 37(1):104-20

10. Lynch JW, Kaplan GA, Shema SJ. Cumulative impact of sustained economic hardship on physical, cognitive, psychological, and social functioning. N Engl J M ed 1997; 337(26):1889-95.

11. Berkman CS, Gurland BJ. The relationship among income, other socioeconomic indicators, and functional level in older persons. J Aging Health 1998; 10(1):81-98.

12. Parker M G, Ahacic K, Thorslund M , Lundberg O. Socioeconomic status and health among elderly people in Sweden. Ann N Y Acad Sci 1999; 896:383-5.

13. Liao Y, McGee DL, Kaufman JS, Cao G, Cooper RS. Socioeconomic status and morbidity in the last years of life. Am J Public Health 1999; 89(4):569-72.

14. Santariano WA. Race, socioeconomic status, and health: a study of age differences in a depressed area. Am J Prev Med 1986; 2:1-5.
15. Kaplan GA, Seeman TE, Cohen RD, Knudsen LP, Guralnik J. Mortality among the elderly in the Alameda Country Study: behavioural and demographic risk factors. Am J Public Health 1987; 77(3):307-12.

16. Feldman JB, M arkuc DM , Kleinman JC, Coroni-Huntley J. National trends in educational differentials in mortality. Am J Epidemiol 1989; 129(5):919-33.

17. HouseJS, Kessler RC, HerzogAR, M ero RP, KinneyAM, Breslow MJ. Age, socioeconomic status and health. Milbank Q 1990; 68:383-411.

18. House JS, Lepkowski JM , Kinney AM, M ero RP, Kessler RC, Herzog AR. The social stratification of aging and health. J Health Soc Behav 1994; 35(3):213-34.

19. Sorlie PD, Backlund E, Keller JB. US mortality by economic, demographic, and social characteristics: the National Longitudinal Mortality Study. Am J Public Health 1995; 85(7):949-56.

20. Elo IT, Preston SH. Educational differentials in mortality: United States, 1979-85. Soc Sci Med 1996; 42(1):47-57.

21. Beckett M. Converging health inequalities in later-life: an artifact of mortality selection. J Health Soc Behav 2000; 41(1):106-19.

22. Lima-Costa M F, Barreto S, Giatti L, U choa E. Desigualdade social e saúde entre idosos brasileiros: um estudo baseado na Pesquisa Nacional por Amostra de D omicílios (PNAD 98). Cad Saúde Pública 2003; 19(3):745-57.

23. Lima-Costa M F, Barreto SM, Firmo JOA, U choa E. Socioeconomic position and health in a popuIation of Brazilian Elderly: The Bambuí Health and Ageing Study (BHAS). Rev Panam Salud Publica 2003; 13(6):387-94.

24. Lima-Costa M FF, Barreto SM , Giatti L. A situação socioeconômica afeta igualmente a saúde de idosos e adultos mais jovens no Brasil? Um estudo utilizando dados da Pesquisa Nacional por Amostra de Domicílios (PNAD 98). Rev C S Col 2002; 7(4):285-96.

25. Brasil. M inistério do Planejamento, O rçamento eGestão. Instituto Brasileiro de Geografia eEstatística. Pesquisa Nacional por Amostra de Domicílios (PNAD 1998). Rio de Janeiro: IBGE; 1998.

26. Brasil. M inistério do Planejamento, Orçamento eGestão. Instituto Brasileiro de Geografia e Estatística. Pesquisa Nacional por Amostra de Domicílios (PNAD 2003). Rio de Janeiro: IBGE; 2003.

27. Paes de Barros R, Henriques R, M endonça R. A estabilidade inaceitável: desigualdade e pobreza no Brasil. Rio de Janeiro: Ipea; 2001. (Texto para Discussão no 800).

28. Lima-Costa M F, Peixoto SV, Matos DL, Firmo JOA, U chôa $E$. A influência de respondente substituto na percepção da saúde em idosos: um estudo baseado na Pesquisa Nacional por Amostras de Domicílios e na coorte de Bambuí (1998 e 2003). Cad Saúde Pública. (No prelo 2006).

29. Hosmer DW, Lemeshow S. Applied logistic regression. New York: Johns Wiley and Sons; 1989.

30. Stata Statistical Software [computer program]. Release 7.0 College Stations 2001. Texas: Stata Corporation; 2001.

Artigo apresentado em 29/03/2006

Aprovado em 24/04/2006

Versão final apresentada em 15/05/2006 